\title{
Carriers of Electricity in the Atmosphere.
}

By Prof. A. M. Tyndall.

THE nature of ions in air has been a subject of study for more than thirty years, but our information is still incomplete and a variety of phenomena still require elucidation. A certain amount of information on the nature of ions in the lower atmosphere may be gained from a study of their motion in an electric field. Except in special cases which rarely arise at ordinary pressures, the motion of an air ion through the air is analogous to the motion of a sphere falling through a viscous liquid. This motion is one of uniform velocity the value of which depends upon the radius of the sphere, the force acting upon it, and the viscosity of the liquid. For a given force and medium, the larger the sphere the slower it moves. This may readily be demonstrated in a syrupy liquid by dropping into it two balls, one of lead and the other of aluminium, their relative sizes having been selected so that they have equal weights in the liquid.

Similarly, ions in air possessing the same electric charge but having different sizes, will move in an electric field at different rates, the larger one travelling slower. There are theoretical grounds for supposing that this factor of size, though not the only one, is of great importance in determining the motion of the ion.

Some interesting effects may be obtained by adding small quantities of an organic vapour to the air. Let us take, for example, the series of normal alcohols of chemical composition $\mathrm{CH}_{3}\left(\mathrm{CH}_{2}\right)_{n} \mathrm{OH}$, where $n$ may be zero or an integer. The molecules of these substances are known to be rod-like in shape, the length of the rod increasing with increase in the carbon content, i.e. with the value of $n$. They are also known to be polar, a property presumably mainly due to the $\mathrm{OH}$ group made up of a positive hydrogen nucleus and a negative atom of oxygen. For the purposes of a rough static model, these molecules may therefore be thought of as having an active head and a more or less inert tail, and they will be attracted to a negative ion with their heads inwards towards the ion and their tails spread out radially. The effective size of the ion will thereby be increased by an amount which depends on the length of the molecules of the particular alcohol added.

Experiments by L. R. Phillips and myself have shown that for a given vapour pressure of alcohol the reduction in mobility of an ion increases rapidly with increase in length of alcohol chain. Thus the highest alcohol (amyl) used in this work, though present as only l part in 300 of air, reduced the mobility of the ordinary negative air ion to about 40 per cent of its normal value. The effect on positive ions is nothing like so marked, though it is observable. This may be attributed to $\mathrm{a}$ weaker bond between the dipole and a positive ion, because the positive end of the dipole cannot approach it so closely.

Substance of a Friday evening discourse delivered at the Royal Institution on $A$ pril 27.
By adding water vapour as well as alcohol vapour to the air, there is now a competition for places at the ion surface, so that short water molecules replace some of the longer alcohol molecules. We should on this view expect a rise in mobility of the ion, and this is observed.

If the molecules are made non-polar by removing the $\mathrm{OH}$ group and making them symmetrical in structure, the tendency to cluster on the ion should be almost entirely removed. This has also been confirmed by experiment, since it has been found that the hydrocarbon, decane, with ten carbon atoms and therefore roughly twice the length of the amyl alcohol molecule, has practically no effect on the motion of the ion in air.

New methods for measuring mobility have been devised with the special purpose of deciding whether all the ions move with the same speed. Considerable resolving power has been achieved. The negative ions appear to be of a single kind in air containing appreciable quantities of vapour. The positive ions over the same range and the negative ions in the presence of small traces of vapour appear to be complex. Certain features present themselves which are still the subject of investigation.

A lecture demonstration of the loading effect of alcohol may be conveniently made by applying a small voltage to an ionisation chamber so that the ionisation current is well below saturation. By blowing alcohol vapour into the chamber the current is reduced to about a half, due to the reduction in mobility of the ions conveying it. The ionisation current may be amplified by a valve method so that it is recorded on a galvanometer.

In the ordinary atmosphere, complicating factors are introduced by the presence of particles of dust, smoke, mist, and other nuclei. A number of small ions will attach themselves to these and will then move so slowly that their contribution to an ionisation current is practically negligible. In the demonstration referred to above, this may be readily shown by blowing tobacco smoke into the chamber, when the ionisation current to all intents ceases. In addition to the production of large ions by the union of small ions with nuclei, large ions may also be produced by the splashing and breaking up of water drops by frictional effects in dust storms and so forth. The presence of these large ions has marked local effects on the potential gradient at the earth's surface and the value of the air-earth current at a given place. Certain fluctuations in these values have been correlated with variations in the number of nuclei present.

When ions of a given sign are dragged through a gas by an electric field, they set the air in motion. With the relatively intense ionisation current from an electrified point this gives rise to the well-known phenomenon of the electric wind. One of the earliest methods of measuring the mobility of ions was based upon a study of this phenomenon. 
Again, if a discharge of this type takes place in a smoky atmosphere, the electric wind assists in carrying the charged smoke particles towards the surrounding surfaces, where the particles stick on impact. In other words, it acts as a smoke precipitator.

To sum up, it may therefore be said that the subject of atmospheric ions has a bearing on at least two important problems at the present time. First, there is the problem of the mode and mechanism of attachment of molecules and ions, linking up with allied problems in the structure of bodies in general; and secondly, there is the wide field of meteorology and the problem of atmospheric electricity in particular.

\section{Natural Steam Power in California.}

By Dr. E. T. Allen and Arthur L. Day,

Geophysical Laboratory, Carnegie Institution of Washington.

F Prince Ginori Conti's remarkable experiments in utilising the potential power in natural steam, the readers of NATURE have been kept informed $(121,59-62 ;$ Jan. 14, 1928). The novelty of his conception and the patience and ingenuity with which it has been pursued to full realisation have attracted much attention among engineers and the public, and people have already the Dutch East Indies one well, bored to a depth of $66 \mathrm{~m}$., showed a pressure (closed) of $4 \frac{1}{2}$ atmospheres and a potential power development of $900 \mathrm{kw}$. Other borings are contemplated in a number of fumarole areas in Java and Sumatra. The Valley of Ten Thousand Smokes, which has been mentioned in this connexion, is much too remote to claim consideration from a commercial viewpoint; besides,

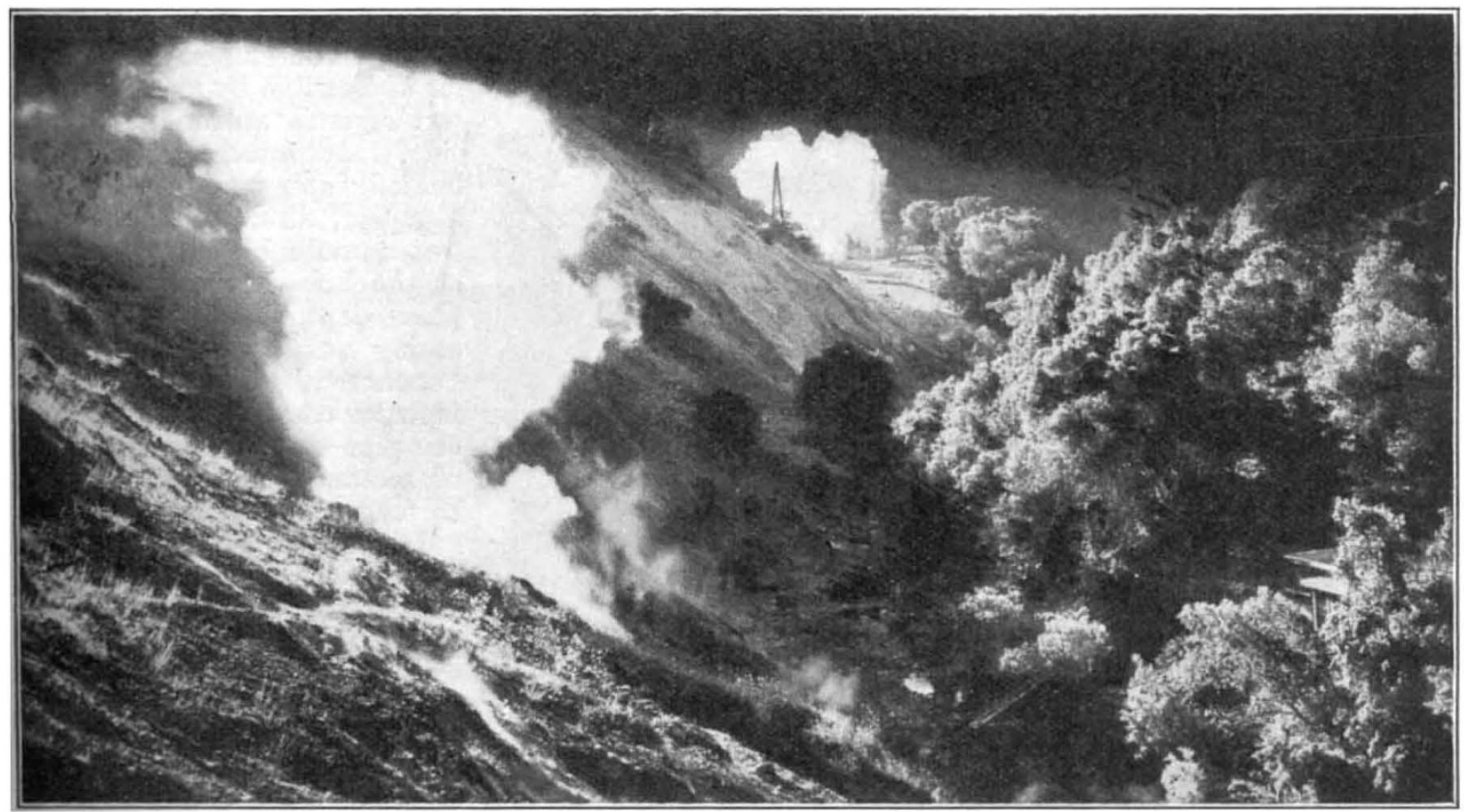

Frg. 1.-Sulphur Creek canyon looking east.

begun to consider the possibility of similar projects elsewhere.

The locality in Tuscany which is the scene of these experiments has long been known as a centre of the boric acid industry, but few have had any definite conception of its character. It is, or rather was before industrial exploitation had modified its appearance, a barren tract covered here and there with very hot steaming springs and vents from which natural steam gushed out in jets of varying size--not infrequently with impressive noise and velocity. Exploration has since brought to light similar regions in other parts of the world, but the Tuscan field still appears to be unusual in the high proportion of its steam output.

Preliminary prospecting for natural steam with the drill in Bolivia and in Oregon has proved unpromising; the flow of steam was too feeble. In the most recent exploration in that region (1923) has proved that a great drop in the surface temperatures has occurred there in less than five years. The Italians have considered, and are perhaps still considering, the sinking of steam wells at Pozzuoli, near Naples, though we have not learned that actual borings have been made there. Recent advices from California inform us that a test hole drilled in Imperial Valley to a depth of 725 feet yielded steam at $175 \mathrm{lb}$. pressure; but the only development known to us that approaches the achievement in Tuscany has been carried out at The Geysers, a place 75 miles north of San Francisco and about 30 miles from the Pacific coast. It lies near the bottom of a deep V-shaped valley enclosed by steep mountain slopes, and reveals its presence to the approaching traveller as a barren stretch of ground from which on cold or damp

No. 3062 , VoL. 122] 